\title{
2018 Reviewer Acknowledgement
}

\author{
Editorial Office of Journal of Clinical and Translational Hepatology
}

We thank the following reviewers for their contribution and support in 2018 .

Mai Ali Abd el Meguid
Egypt
Sherief Abd-Elsalam
Egypt
Avin Aggarwal
United States
Nobuhisa Akamatsu
Japan
Kawtar Alkhalloufi
United States
Khaled Amer
Egypt
Masahiro Arai
Japan
Leon Averbukh
United States
Gyorgy Baffy
United States
Mahmoud Mohamed Bahgat
Egypt
Jodie A Barkin
United States
Roopjeet Bath
United States
Mattia Bellan
Italy
Christian Benzing
Germany
Marina Berenguer
Spain
Ines Bilic-Curcic
Croatia
Uimothy Billiar
United States

DOI: $10.14218 /$ JCTH.2018.000RA

\author{
Han Bing \\ China \\ Ilka Bion \\ Brazil \\ John W Birk \\ United States \\ Claus Thomas Bock \\ Germany \\ Daniel Bodek \\ United States \\ Peter Buch \\ United States \\ Chalermrat Bunchorntavakul \\ Thailand \\ wenqing Cao \\ United States \\ Naichaya Chamroonkul \\ Thailand \\ Roger W. Chapman \\ United Kingdom \\ Phunchai Charatcharoenwitthaya \\ Thailand \\ Limin Chen \\ Canada \\ Yongpeng Chen \\ China \\ Chunmei Chen \\ China \\ Jun Chen \\ China \\ John Chiang \\ United States \\ José Artur Chies \\ Brazil \\ Gourdas Choudhuri \\ India \\ Ashok CHOUDHURY \\ India
}

\author{
S. Sylvester Darvin \\ India \\ Jianqiang Ding \\ China \\ Xingrong Du \\ United States
}

\section{Atanu Kumar Dutta}

India

Marko Duvnjak

Croatia

Cumali Efe

Turkey

Mostafa Kamel El-Awady

Egypt

Mohamed A. El-Guindi

Egypt

Gülsüm Ozlem Elpek

Turkey

Saleh Elwir

United States

Yuchen Fan

China

Jiangao Fan

China

Silvia Fargion

Italy

Fatemeh Farshadpour

Iran (Islamic Republic of)

Eduardo Fernández-Martínez

Mexico

Catherine Frenette

United States

Artin Galoosian

United States

\section{Antonio Giorgio}

Italy

Mohammad Mehdi Gouya Iran (Islamic Republic of) 
Severin O. Gudima

United States

Andrew John Gunn

United States

Birendra Prasad Gupta

Nepal

Ying Han

China

Kazuhiko Hayashi

Japan

Noha Helal

Egypt

Jeong Heo

Korea, Republic of

Cheng-Maw Ho

Taiwan

Yunyi Hong

China

Johannes Hov
Norway

Christine Hsu

United States

Yoshio Ijiri

Japan

Hartmut Jaeschke

United States

Abdurrahman Kadayifci

United States

sandeep kumar karn

China

Lindsey Kennedy

United States

AM Khairy

Egypt

Tawfik khoury

Israel

Hyunseok Kim

United States

John Koskinas

Greece

Somashekar G Krishna

United States

Karen Krok

United States

Anand kulkarni

India

Ravikant Kumar

India

Ashish Kumar

India
Kwang-Ho Lee

Korea, Republic of

Xiaocong Liu

United States

Chenghai Liu

China

Bing Liu

China

Shuang Liu

China

Stephen Locarnini

Australia

Lungen Lu

China

Stefan Luth

Germany

Philipp Lutz

Germany

Shaina Lynch

United States

Michelle Ma

United States

Mojtaba mafi

Iran (Islamic Republic of)

Matthew McMillin

United States

Kamalika Moulick

United States

Pradeep Naik

India

Seyedehsan Navabi

United States

James S Park

United States

Yanyan Peng

China

M. Pokorska-Śpiewak

Poland

Xingshun Qi

China

Cristiano Quintini

United States

Atoosa Rabiee

United States

Alok Ranjan

United States

Ciro Romano

Italy

Manuel Romero-Gómez

Spain
Lionel Rostaing

France

Behnam Saberi

United States

Hidetsugu Saito

Japan

Ghada Salum

Egypt

Keisaku Sato

United States

Yehia M Shaker

Egypt

A. M. J. Shapiro

Canada

Hao Shi

United States

Shivaram Prasad Singh

India

Surajit Sinha

United States

Coleman Smith

United States

Martina Smolic

Croatia

Shanthi Srinivasan

United States

Eike Steinmann

Germany

Qingfeng Sun

China

Ashraf Tabll

Egypt

Mamoru Takenaka

Japan

Rahul Pundlik Talele

India

Giovanni Tarantino

Italy

Georgios Tsoulfas

Greece

Lucija Virovic-Jukic

Croatia

Lili Wang

United States

Benjamin I Woolbright

United States

Yongning Xin

China

Manfung Yuen

Hong Kong 
2018 Reviewer Acknowledgement

Rong Zhang

United States

Peng Zhang

United States

Qingzhan Zhang

United States
Yuexin Zhang China

Lanjing Zhang

United States

Hui Zhang

United States
Jingmin Zhao

China

Jianhong Zhong

China 